\title{
ON THE NUMBER OF MODULI OF EXTENDABLE CANONICAL CURVES
}

\author{
CIRO CILIBERTO*AND ANGELO FELICE LOPEZ
}

\begin{abstract}
Let $C \subset \mathbb{P}^{g-1}$ be a canonical curve of genus $g$. In this article we study the problem of extendability of $C$, that is when there is a surface $S \subset \mathbb{P}^{g}$ different from a cone and having $C$ as hyperplane section. Using the work of Epema we give a bound on the number of moduli of extendable canonical curves. This for example implies that a family of large dimension of curves that are cover of another curve has general member nonextendable. Using a theorem of Wahl we prove the surjectivity of the Wahl map for the general k-gonal curve of genus $g$ when $k=5, g \geq 15$ or $k=6, g \geq 13$ or $k \geq 7, g \geq 12$.
\end{abstract}

\section{$\S 1$. Introduction}

Let $C \subset \mathbb{P}^{g-1}$ be a smooth irreducible canonical curve of genus $g$. When $C$ has general moduli (and $\mathrm{g}$ is not too small) the fact that the moduli space of curves is of general type suggests that $C \subset \mathbb{P}^{g-1}$ is nonextendable, i.e. that there is no surface $S \subset \mathbb{P}^{g}$ different from a cone and having $C$ as hyperplane section. This turns out to be indeed the case for $g=10$ or $g \geq 12$ : first Wahl introduced the Gaussian-Wahl map $\Phi_{\omega_{C}}: \bigwedge^{2} H^{0}\left(\omega_{C}\right) \rightarrow H^{0}\left(\omega_{C}^{3}\right)$ and proved that a curve $C$ cannot lie on a K3 surface if $\Phi_{\omega_{C}}$ is surjective [W1]; in fact Wahl proved this by showing that the cone over $C$ in $\mathbb{P}^{g}$ has no infinitesimal nonconical deformations, thus that $C \subset \mathbb{P}^{g-1}$ is nonextendable when $\Phi_{\omega_{C}}$ is surjective (for another version see F.L. Zak [Z] and L'vovsky $[\mathrm{Lv}])$. Secondly Ciliberto, Harris and Miranda ([CHM], reproved by many others $[\mathrm{V} 2],[\mathrm{P}],[\mathrm{L}])$ showed that the Wahl map is surjective for a general curve of genus 10 or at least 12 . From the above facts two questions naturally arise: 1) is there some kind of converse to Wahl's theorem? 2) how special are extendable canonical curves?

In 1995 Wahl [W3] proved a beautiful theorem that addresses the first question: suppose that $C \subset \mathbb{P}^{g-1}$ satisfies $(*) H^{1}\left(\mathfrak{I}_{C}^{2}(t)\right)=0$ for every

Received October 15, 1999.

2000 Mathematics Subject Classification: Primary 14H10. Secondary 14J10, 14J26.

*Research partially supported by the MURST national project "Geometria Algebrica"; the authors are members of GNSAGA of CNR. 
$t \geq 3$ and, moreover, $\Phi_{\omega_{C}}$ is not surjective; then $C$ is extendable. In the same article Wahl gave some indication on the curves satisfying the above condition: $H^{1}\left(\mathfrak{I}_{C}^{2}(3)\right) \neq 0$ for a tetragonal curve, while $(*)$ holds for a general pentagonal curve. Moreover he conjectured that $(*)$ holds for large $g$ and for any curve with Clifford index at least 3 . The importance of Wahl's conjecture lies, besides the natural questions of extendability, also in its relation with one of the most interesting open problems about algebraic curves, i.e. Green's conjecture: Cliff $C>p$ if and only if $\mathrm{C}$ satisfies property $\left(N_{p}\right)$, that is $C \subset \mathbb{P}^{g-1}$ is projectively normal, its ideal is generated by quadrics and the syzygies are generated by linear polynomials up to the p-th module [G]. This conjecture holds for $p \leq 2$ ([S], [V1]) and for low values of $g$. As remarked by Wahl [W3] if Green's conjecture holds for $p=3$ then $H^{1}\left(\mathfrak{I}_{C}^{2}(4)\right)=0$ for every curve with Cliff $C>3$.

Our investigation on the above subject started with the simple observation that if one could find a nonextendable canonical curve $C$ with nonsurjective Wahl map, large genus and Clifford index at least 3, then, by Wahl's theorem [W3, Theorem 7.1], this curve would be a counterexample to Wahl's conjecture. Of course this gives $H^{1}\left(\Im_{C}^{2}(t)\right) \neq 0$ for some $t \geq 3$ (in fact $t=3$ or 4 ) and then, to get a possible counterexample to Green's conjecture, one should check if among such curves there are curves $C$ with Cliff $C>3$ and $H^{1}\left(\Im_{C}^{2}(4)\right) \neq 0$.

Let now $C \subset \mathbb{P}^{g-1}$ be an extendable canonical curve and $S \subset \mathbb{P}^{g}$ a surface different from a cone and having $C$ as hyperplane section. It is well known that, if $S$ is smooth, then it is a K3 surface. When $S$ is singular the nice work of Epema [E1], [E2] gives a complete description of a minimal desingularization $\widetilde{S}$ of $S$. In particular he proves that either $\widetilde{S}$ is a minimal $\mathrm{K} 3$ surface or it is ruled and $\operatorname{dim}\left|-K_{\widetilde{S}}\right|=0$. We use these facts and some projective techniques to give a bound on the number of blow-ups needed in the ruled case and therefore on the number of moduli on which such surfaces can depend. As a consequence we get a bound on the number of moduli of extendable canonical curves, as follows. Consider the following loci in $\mathcal{M}_{g}$ :

$W_{1}=\left\{[C] \in \mathcal{M}_{g}:\right.$ Cliff $C \geq 1$ and $C$ is extendable in its canonical embedding $\}$, and, for $2 \leq \alpha \leq 4$, $W_{\alpha}=\left\{[C] \in W_{\alpha-1}: C\right.$ is not an $\alpha: 1$ cover of another curve $\}$.

Then 
THEOREM (1.1). For $g \geq 12$ the following bounds hold:

(i) $\operatorname{dim} W_{\alpha} \leq \max \{g+19,2 g-1\}$ for $\alpha=1,2$;

(ii) $\operatorname{dim} W_{3} \leq \frac{5}{3} g+12$;

(iii) $\operatorname{dim} W_{4} \leq \frac{3}{2} g+14$.

Now if one wants to apply this theorem to get a counterexample to Wahl's conjecture it is enough to produce a family of curves of dimension exceeding the bounds of Theorem (1.1) and whose general element has large genus, Clifford index at least 3 and nonsurjective Wahl map. As of now though all known examples of families of curves with nonsurjective Wahl map have smaller dimension, except for double and (some) triple covers (see Proposition (4.1)). This is also related to the open problem of giving a bound for the dimension of the components of the Wahl locus, that is the locus of curves with nonsurjective Wahl map. In section three we will also show that a general $p: 1$ cover of a general curve is, in most cases, nonextendable (Proposition (3.1)), but even for the simplest cases of a general triple cover of a curve of genus 2 or $5: 1$ cover of an elliptic curve it is not known whether the Wahl map is surjective or not. This and related open questions will be discussed in section four. In any event one nice consequence of Theorem (1.1) is that it allows to settle the question of the surjectivity of the Wahl map for a general k-gonal curve: Ciliberto and Miranda [CM], Wahl [W2] proved that it is not surjective for $k=2,3$, Brawner [B1], [B2] showed that the same is true for $k=4$ (and 3). The behavior instead changes for $k \geq 5$.

THEOREM (1.2). Let $C$ be the general $k$-gonal curve of genus $g \geq 12$. Then the Wahl map $\Phi_{\omega_{C}}$ is surjective as soon as

(i) $k=5$ and $g \geq 15$;

(ii) $k=6$ and $g \geq 13$;

(iii) $k \geq 7$.

It should be noted that a special case of (ii) above (when $g \equiv 2(\bmod$ 5)) was shown by Wahl in [W2]. 


\section{$\S 2$. Surfaces with canonical hyperplane section}

In this section we intend to investigate the properties of surfaces with canonical hyperplane sections, giving particular emphasis on the number of moduli on which they depend.

Definition (2.1). A surface $S \subset \mathbb{P}^{g}$ with canonical hyperplane sections is an irreducible nondegenerate surface such that its general hyperplane section $C=S \cap H \subset \mathbb{P}^{g-1}$ is a smooth canonical curve of genus $g$ and $S$ is not a cone over $C$.

A very nice description of many properties of these surfaces is given in Epema's thesis [E1], [E2]. We start by recalling some of the facts proved there.

Proposition (2.2). Let $S \subset \mathbb{P}^{g}$ be a surface with canonical hyperplane sections and let $p: \widetilde{S} \rightarrow S$ be the minimal desingularization. Then

(2.3) $S$ is projectively normal;

(2.4) $\widetilde{S}$ is either a minimal K3 surface or a ruled surface;

(2.5) $\operatorname{dim}\left|-K_{\widetilde{S}}\right|=0$.

Proof. (2.3) and (2.4) are proved in [E1, Proposition I.3.1]. (2.5) is in [E1, Corollary I.5.4].

Let $S \subset \mathbb{P}^{g}$ be a surface with canonical hyperplane sections, $p: \widetilde{S} \rightarrow S$ be the minimal desingularization and suppose that $\widetilde{S}$ is a ruled surface. Set $q=q(\widetilde{S})$ and let $S_{0}$ be a minimal model for $\widetilde{S}$. Then either $S_{0}=\mathbb{P}^{2}$ or there exist a smooth irreducible curve $\Gamma$ of genus $q$ and a rank two (normalized) vector bundle $E$ over $\Gamma$ such that $S_{0}=\mathbb{P} E \stackrel{\pi}{\rightarrow} \Gamma$. We let $L=$ $\mathcal{O}_{S}(C), \widetilde{L}=p^{*} L=\mathcal{O}_{\widetilde{S}}(\widetilde{C}), \pi_{0}: \widetilde{S} \rightarrow S_{0}$ the natural morphism, $V_{0} \subset H^{0}\left(L_{0}\right)$ the corresponding linear system of dimension $g$ on $S_{0}$. The morphism $\pi_{0}$ : $\widetilde{S} \rightarrow S_{0}$ is the composition of $h$ blowing ups at base points of $V_{0}$ and possibly at infinitely near points [E1]. We let $E_{i}$ be the corresponding exceptional divisor on $\widetilde{S}$ and $r_{i} \geq 1$ integers such that

$$
\widetilde{L}=\mathcal{O}_{\widetilde{S}}(\widetilde{C})=\pi_{0}^{*} L_{0}\left(-\sum_{i=1}^{h} r_{i} E_{i}\right)
$$

Moreover, when $S_{0} \neq \mathbb{P}^{2}$, let $a \in \mathbb{Z}, \Delta \in \operatorname{Pic} \Gamma$ such that $L_{0}=\mathcal{O}_{S_{0}}\left(a C_{0}+\right.$ $\left.\pi^{*}(\Delta) f\right)$ where $C_{0}$ is a section of $\pi, f$ a fiber and $C_{0}^{2}=-e=\operatorname{deg} E$. Note 
that then $S$ is ruled over $\Gamma$ by curves of degree $a$ in $\mathbb{P}^{g}$. Again we recall from $[\mathrm{E} 1]$ the following facts.

Proposition (2.6). Suppose $S_{0} \neq \mathbb{P}^{2}$. After possibly replacing $S_{0}$ by another minimal model we can assume:

(2.7) $E \cong \mathcal{O}_{\Gamma} \oplus \mathcal{O}_{\Gamma}(D)$;

(2.8) $\Delta \sim-a D$;

(2.9) either $D \sim-K_{\Gamma}$ or $h \geq 1$ and $e>2 q-2$;

(2.10) $r_{i} \leq a-1$;

(2.11) $\sum_{i=1}^{h} r_{i}=a(e-2 q+2)$;

$g=1+\frac{1}{2} a^{2} e-\frac{1}{2} \sum_{i=1}^{h} r_{i}^{2}$

(2.13) $1+a^{2}(q-1)+\frac{1}{2} a(e-2 q+2) \leq g \leq 1+\frac{1}{2} a^{2} e-\frac{1}{2} a(e-2 q+2)$;

(2.14) $h \leq \frac{2}{a-1}\left[g-1-a^{2}(q-1)\right]$ for $a \geq 2$.

Proof. With the exception of (2.14), this is the content of [E1, Proposition III.1.4]. To see (2.14) set, for $1 \leq j \leq a-1, h_{j}=\sharp\left\{i: r_{i}=j\right\}$. By $(2.11)$ and $(2.12)$ we get $g=1+a^{2}(q-1)+\frac{1}{2} \sum_{i=1}^{h}\left(a r_{i}-r_{i}^{2}\right)$. Now $\sum_{i=1}^{h}\left(a r_{i}-r_{i}^{2}\right)=\sum_{j=1}^{a-1} h_{j}\left(a j-j^{2}\right) \geq(a-1) h$ as $a j-j^{2} \geq a-1$.

Observe that in any case $\operatorname{dim}\left|-K_{S_{0}}\right| \geq 0$. We need the following (sharp) estimate.

Proposition (2.15). Suppose e $>2 q-2$. Let $\Gamma$ be a smooth irreducible curve of genus $q, D$ a divisor on $\Gamma$ of degree $-e, S_{0}=\mathbb{P}^{2}$ or $\mathbb{P}\left(\mathcal{O}_{\Gamma} \oplus \mathcal{O}_{\Gamma}(D)\right)$. Then

$$
\operatorname{dim}\left|-K_{S_{0}}\right|-\operatorname{dimAut} S_{0} \leq\left\{\begin{array}{cl}
1 & \text { if } q=0 \text { and } S_{0}=\mathbb{P}^{2} \\
2 & \text { if } q=0 \text { and } S_{0} \neq \mathbb{P}^{2} \\
-1 & \text { if } q=1 \\
-2 q & \text { if } q \geq 2, e \geq 4 q-3 \\
-\frac{1}{2} e-1 & \text { if } q \geq 2, e \leq 4 q-4
\end{array}\right.
$$


Proof. The result being obvious if $S_{0}=\mathbb{P}^{2}$, we suppose otherwise. We have $-K_{S_{0}} \sim 2 C_{0}-\pi^{*}\left(K_{\Gamma}+D\right) f$, hence $h^{0}\left(S_{0}, \mathcal{O}_{S_{0}}\left(-K_{S_{0}}\right)\right)=h^{0}\left(\Gamma, S^{2}\left(\mathcal{O}_{\Gamma} \oplus\right.\right.$ $\left.\left.\mathcal{O}_{\Gamma}(D)\right) \otimes \mathcal{O}_{\Gamma}\left(-K_{\Gamma}-D\right)\right)=h^{0}\left(\mathcal{O}_{\Gamma}\left(-K_{\Gamma}-D\right)\right)+h^{0}\left(\mathcal{O}_{\Gamma}\left(-K_{\Gamma}+D\right)\right)+$ $h^{0}\left(\mathcal{O}_{\Gamma}\left(-K_{\Gamma}\right)\right)$. If $q=0$ we deduce

$$
\operatorname{dim}\left|-K_{S_{0}}\right|=\left\{\begin{array}{ll}
e+5 & \text { if } e \geq 3 \\
8 & \text { if } e \leq 2
\end{array} .\right.
$$

If $q=1$ we get

$$
\operatorname{dim}\left|-K_{S_{0}}\right|=e .
$$

When $q \geq 2$ we have, by Clifford's theorem,

$$
\operatorname{dim}\left|-K_{S_{0}}\right| \leq \begin{cases}e-3 q+2 & \text { if } h^{1}\left(\mathcal{O}_{\Gamma}\left(-K_{\Gamma}-D\right)\right)=0 \\ \frac{1}{2} e-q+1 & \text { if } h^{1}\left(\mathcal{O}_{\Gamma}\left(-K_{\Gamma}-D\right)\right) \neq 0\end{cases}
$$

Let us now compute $\operatorname{dim} A u t S_{0}$. For $q \geq 1$, since $h^{0}\left(E \otimes E^{*}\right)=h^{0}\left(\mathcal{O}_{\Gamma}(-D)\right)$ $+2 h^{0}\left(\mathcal{O}_{\Gamma}\right)+h^{0}\left(\mathcal{O}_{\Gamma}(D)\right) \geq e-q+3$, by $(2.17)$, (2.18) we get the bound. When $q=0$ and hence $S_{0}=\mathbb{F}_{e}$ it is well known that

$$
\operatorname{dimAut} S_{0}=h^{0}\left(T_{\mathbb{F}_{e}}\right)= \begin{cases}e+5 & \text { if } e \geq 1 \\ 6 & \text { if } e=0\end{cases}
$$

and this, together with $(2.16)$, gives the required bound.

We can now give our estimate on the number of moduli of surfaces with canonical hyperplane sections.

Proposition (2.19). Let $S \subset \mathbb{P}^{g}$ be a surface whose general hyperplane section is a smooth canonical curve $C \subset \mathbb{P}^{g-1}$. Let $p: \widetilde{S} \rightarrow S$ be the minimal desingularization and suppose that $\widetilde{S}$ is a ruled surface obtained from a minimal model $S_{0}=\mathbb{P}\left(\mathcal{O}_{\Gamma} \oplus \mathcal{O}_{\Gamma}(D)\right)$ by $h$ blow-ups. Let $q=q\left(S_{0}\right), e=-\operatorname{deg} D$. Suppose $S_{0}$ fixed and let $\Sigma$ be a component of the family of such surfaces $\widetilde{S}$. Then the following bounds hold:

$$
\operatorname{dim} \Sigma \leq \max \left[0,\left\{\begin{array}{ll}
h-2 q & \text { if } q \geq 2, e \geq 4 q-3 \\
h-\frac{1}{2} e-1 & \text { if } q \geq 2, e \leq 4 q-4 \\
h-1 & \text { if } q=1
\end{array}\right]\right.
$$

(2.21) $\operatorname{dim} \Sigma \leq\left\{\begin{array}{ll}\frac{2}{3} g+12 & \text { if } C \text { is not trigonal } \\ \frac{1}{2} g+14 & \text { if } C \text { is not tetragonal }\end{array}\right.$ and $q=0$. 
Proof. By (2.5) we have $\operatorname{dim}\left|-K_{\widetilde{S}}\right|=0$ hence $\widetilde{S}$ is obtained from $S_{0}$ by blowing up $h^{\prime} \leq h$ points so that the corresponding surface $S^{\prime}$ satisfies $\operatorname{dim}\left|-K_{S^{\prime}}\right|=0$ and then by further blowing up $h-h^{\prime}$ points on the unique effective anticanonical divisor $-K_{S^{\prime}}$. Therefore either

$$
\operatorname{dim} \Sigma \leq \operatorname{dim}\left|-K_{S_{0}}\right|+h-\operatorname{dimAut} S_{0}
$$

when the left hand side is not negative, or $\operatorname{dim} \Sigma=0$. The bound (2.20) follows then by Proposition (2.15) unless $e=2 q-2$. In the latter case by (2.9) we have $h=0, \widetilde{S}=S_{0}$ and $\operatorname{dim} \Sigma=0$. Now suppose $q=0$. The result in this case is a consequence of (2.22), Proposition (2.15) and

$$
h \leq\left\{\begin{array}{ll}
\frac{2}{3} g+2+K_{S_{0}}^{2} & \text { if } C \text { is not trigonal } \\
\frac{1}{2} g+4+K_{S_{0}}^{2} & \text { if } C \text { is not tetragonal }
\end{array} .\right.
$$

To see (2.23) let $Z \in\left|-K_{\widetilde{S}}\right|$ be the unique effective anticanonical divisor. The exact sequence

$$
0 \rightarrow \mathcal{O}_{\widetilde{S}}\left(K_{\widetilde{S}}\right) \rightarrow \mathcal{O}_{\widetilde{S}} \rightarrow \mathcal{O}_{Z} \rightarrow 0
$$

and the hypotheses $H^{0}\left(\mathcal{O}_{\widetilde{S}}\left(K_{\widetilde{S}}\right)\right)=H^{1}\left(\mathcal{O}_{\widetilde{S}}\left(K_{\widetilde{S}}\right)\right)=0$ imply that $h^{0}\left(\mathcal{O}_{Z}\right)=$ 1 and Supp $Z$ is connected. Therefore $p$ contracts $Z$ to a point $x \in S$. Let $\pi_{x}: S \rightarrow F \subset \mathbb{P}^{g-1}$ be the projection, $F$ its image. As $S$ is not a cone $\pi_{x}$ is generically one to one and $F$ is a surface. Moreover since $\mathcal{O}_{\widetilde{S}}(\widetilde{C}) \otimes \mathcal{O}_{Z} \cong \mathcal{O}_{Z}$ we get $h^{0}\left(\mathcal{O}_{\widetilde{S}}(\widetilde{C}-Z)\right)=g$, hence $\operatorname{deg} F=(\widetilde{C}-Z)^{2}=2 g-2-h+K_{S_{0}}^{2}$. By the exact sequence

$$
0 \rightarrow \mathfrak{I}_{S / \mathbb{P}^{g}}(1) \rightarrow \mathfrak{I}_{S / \mathbb{P}^{g}}(2) \rightarrow \mathfrak{I}_{C / \mathbb{P}^{g-1}}(2) \rightarrow 0
$$

and (2.3) we get an isomorphism $H^{0}\left(\mathfrak{I}_{S / \mathbb{P}^{g}}(2)\right) \rightarrow H^{0}\left(\mathfrak{I}_{C / \mathbb{P}^{g-1}}(2)\right)$ and by [SD, (7.10)], $S$ is intersection of quadrics, as $C$ is not trigonal. Suppose first $h>\frac{2}{3} g+2+K_{S_{0}}^{2}$; then $\operatorname{deg} F<\frac{4}{3} g-4$, hence by $[\mathrm{H}] F$ is ruled by lines and therefore $S$ is ruled by lines or conics. But $S$ is rational, hence $C$ is hyperelliptic, a contradiction. Similarly if $C$ is not tetragonal and $h>\frac{1}{2} g+4+K_{S_{0}}^{2}$ we have $\operatorname{deg} F<\frac{3}{2} g-6$, hence by [H] $F$ is ruled by lines or conics and $S$ is ruled (rationally) by curves of degree up to 4 . But then $C$ is tetragonal. 


\section{§3. Nonextendable canonical curves}

Given the bound on the number of moduli of surfaces that extend a canonical curve $C \subset \mathbb{P}^{g-1}$ proved in section two, we can now proceed to construct many examples of nonextendable canonical curves.

We start with some simple but interesting examples (see also section four). Given three integers $g, p$ and $\gamma$ such that $p \geq 3, \gamma \geq 0, \gamma \geq 1$ if $p=3$, and $g>\max \left\{11,3 p-3+p \gamma, a\left(\frac{\gamma}{a-1}+1\right) p+1,2 \leq a \leq 4\right\}$, we define a function $\phi(g, p, \gamma)$ that will take care of all the inequalities we will need to satisfy. Set

$$
\begin{aligned}
& \phi(g, p, \gamma) \\
& = \begin{cases}\frac{5}{3} g+12 & \text { if } p=4, \gamma=0 \\
2 g-1 & \text { if } p=3, \gamma=1, g \geq 30 \\
\frac{5}{3} g+\frac{1}{3} & \text { if } p=4, \gamma=1, g \geq 82 \\
2 g-7 \gamma+5 & \text { if } p=3, \gamma \geq 2, g>\max \{15 \gamma-14,14 \gamma+18\} \\
2 g-6 \gamma+5 & \text { if } p=3, \gamma \geq 2,12 \gamma+18 \leq g \leq 15 \gamma-14 \\
\frac{5}{3} g-\frac{26}{3} \gamma+7 & \text { if } p=4, \gamma \geq 2, g>\max \{52 \gamma+42,28 \gamma-27\} \\
\frac{5}{3} g-\frac{23}{3} \gamma+7 & \text { if } p=4, \gamma \geq 2,46 \gamma+42 \leq g \leq 28 \gamma-27 \\
\frac{3}{2} g+14 & \text { otherwise. }\end{cases}
\end{aligned}
$$

Proposition (3.1). For $g, p$ and $\gamma$ as above let $W \subset \mathcal{M}_{g}$ be a subvariety whose general element is represented by a curve $C$ such that:

(i) $C$ has a noncomposite morphism $\pi: C \rightarrow X$ of degree $p$, where $X$ is a smooth curve of genus $\gamma$;

(ii) $X$ does not have morphisms of degree at least two onto a curve of genus $q$ for $1 \leq q \leq \frac{\gamma+1}{2}$;

(iii) $\operatorname{dim} W>\phi(g, p, \gamma)$.

Then $\operatorname{Cliff}(C) \geq 3$ (except when $\gamma=0, p=4$ ) and $C$ is nonextendable in its canonical embedding. 
Proof. We first deal with the gonality of $C$. We claim that

$$
\operatorname{gon}(C) \geq \begin{cases}4 & \text { if } \gamma=0, p=4 \\ 5 & \text { otherwise }\end{cases}
$$

Suppose $\operatorname{gon}(C)=d$. By (i) if the morphism $C \rightarrow X \times \mathbb{P}^{1}$ is not birational we have, for some $m \geq 1, d=m p$ and $X$ has an $m: 1$ map onto $\mathbb{P}^{1}$, hence $d \geq 4$ with equality only if $\gamma=0, p=4$. If the morphism $C \rightarrow X \times \mathbb{P}^{1}$ is birational we get $g \leq(p-1)(d-1)+\gamma p$, therefore it cannot be $d \leq 4$. As $g$ is large we have $\operatorname{Cliff}(C) \geq 3$ (except when $\gamma=0, p=4$ ) by [Ma]. Suppose now $C \subset \mathbb{P}^{g-1}$ is the hyperplane section of a surface $S \subset \mathbb{P}^{g}$. Let $p: \widetilde{S} \rightarrow S$ be the minimal desingularization. If $\widetilde{S}$ is a minimal K3 surface we have $\operatorname{dim} W \leq g+19$, contradicting (iii). Otherwise by (2.4) and (2.6) $\widetilde{S}$ is a ruled surface obtained from a minimal model $S_{0}$ (which is either $\mathbb{P}\left(\mathcal{O}_{\Gamma} \oplus \mathcal{O}_{\Gamma}(D)\right)$ or $\left.\mathbb{P}^{2}\right)$ by $h$ blow-ups. Let $q=q\left(S_{0}\right), e=-\operatorname{deg} D$ and $S$ is ruled by curves of degree $a \geq 2$ (if $a=1$, then $S$ is a cone). If $q=0$ we get by $(2.21)$

$$
\operatorname{dim} W \leq \begin{cases}\frac{5}{3} g+12 & \text { if } \gamma=0, p=4 \\ \frac{3}{2} g+14 & \text { otherwise }\end{cases}
$$

again contradicting (iii). We deal now with the case $q \geq 1$. By Proposition (2.19) we have

$\operatorname{dim} W \leq 3 q-3+\eta_{q}+q+g+\max \left[0,\left\{\begin{array}{ll}h-2 q & \text { if } q \geq 2, e \geq 4 q-3 \\ h-\frac{1}{2} e-1 & \text { if } q \geq 2, e \leq 4 q-4 \\ h-1 & \text { if } q=1\end{array}\right]\right.$ where $\eta_{q}=\left\{\begin{array}{ll}0 & \text { if } q \geq 2 \\ 1 & \text { if } q=1\end{array}\right.$. As $e \geq 2 q-2$, we deduce the following bound:

$$
\operatorname{dim} W \leq g+h+ \begin{cases}2 q-3 & \text { if } q \geq 2, e \geq 4 q-3 \\ 3 q-3 & \text { if } q \geq 2, e \leq 4 q-4 \\ 1 & \text { if } q=1\end{cases}
$$

We have also the general bound, given by the number of ramification points,

$$
\operatorname{dim} W \leq 2 g-2-(2 a-3)(q-1)+\eta_{q}
$$

Suppose first $(a, q) \neq(p, \gamma)$. Then by (i) and (ii) the induced map $C \rightarrow$ $X \times \Gamma$ is birational, hence we get the following estimates:

$$
g \leq(a-1)(p-1)+a q+p \gamma
$$




$$
g \leq a\left(\frac{\gamma}{a-1}+1\right) p+1 \text { (this follows from (3.5) and (2.13)). }
$$

By (3.6) and the hypothesis on $g$ it must be $a \geq 5$ hence (2.14) and (3.3) contradict (iii). When $(a, q)=(p, \gamma)$ use again $(2.13),(3.3)$ and (2.14) (and (3.4) if $p=3, \gamma=1)$. Again we get $\operatorname{dim} W \leq \phi(g, p, \gamma)$.

Remark (3.7). The Proposition applies for example to the family $W$ of all curves that are cover of degree $p$ of some curve of genus $\gamma \geq 2$ as (ii) holds for a general curve [ACGH, VIII.C-6].

The methods used to prove the above Proposition also apply to show our main result.

Proof of Theorem (1.1). Let $C$ be a general element of a component of $W_{\alpha}$, so that $C \subset \mathbb{P}^{g-1}$ is hyperplane section of a surface $S \subset \mathbb{P}^{g}$. Let $p: \widetilde{S} \rightarrow S$ be the minimal desingularization. If $\widetilde{S}$ is a minimal K3 surface we have $\operatorname{dim} W_{\alpha} \leq g+19$. Otherwise, with the same notation of the proof of Proposition (3.1), $\widetilde{S}$ is a ruled surface obtained from a minimal model by $h$ blow-ups. By (3.4) we get $\operatorname{dim} W_{\alpha} \leq 2 g-1$ if $\alpha=1,2$. Suppose then $\alpha=3,4$. If $q=0$ we get by $(2.21)$

$$
\operatorname{dim} W_{\alpha} \leq\left\{\begin{array}{ll}
\frac{5}{3} g+12 & \text { if } \alpha=3 \\
\frac{3}{2} g+14 & \text { if } \alpha=4
\end{array} .\right.
$$

For $q \geq 1$, as $C$ is a cover of degree $a$ of another curve, we have $a \geq \alpha+1$. Therefore we conclude applying (3.3) and (2.14).

We now consider general $k$-gonal curves.

Proof of Theorem (1.2). By Wahl's result [W3, Corollary 5.4] we know that a general pentagonal curve $C_{0}$ of genus $g \geq 8$ satisfies the vanishing $H^{1}\left(\Im_{C_{0}}^{2}(t)\right)=0$ for every $t \geq 3$. Let now $C$ be a curve as in the hypotheses of the Theorem. Then $C$ is $k$-gonal for $k \geq 5$, and, as it is well known, it specializes to $C_{0}$. Therefore we also have $H^{1}\left(\mathfrak{I}_{C}^{2}(t)\right)=0$ for every $t \geq 3$, by semicontinuity. Hence Theorem (1.1) and Wahl's theorem [W3, Theorem 7.1] give that the Wahl map of $C$ is surjective as long as the locus $\mathcal{M}_{g, k}^{1}$ of $k$-gonal curves has dimension larger than $\max \{g+19,2 g-1\}$. Now it is well known that $\operatorname{dim} \mathcal{M}_{g, k}^{1}=2 g-5+2 k$ if $k \leq \frac{1}{2} g+1[\mathrm{AC}]$, hence we get the theorem. 
Remark (3.8). The same count of parameters shows that a general tetragonal curve of genus $g \geq 17$ is nonextendable, even though its Wahl map is not surjective. This can be probably proved also by the methods of [W4].

Remark (3.9). We recall that a standard consequence of Theorem (1.2) is the surjectivity of the Gaussian maps $\Phi_{\omega_{C}, L}$ on a general $k$-gonal curve $C$ as in (1.2), where $L$ is a line bundle on $C$ that is either general of degree at least $2 g-2+k$ or any line bundle of degree at least $3 g-1+k$ (see [L, proof of Corollary 1.7]).

\section{§4. Examples and open problems}

As already observed in the introduction despite all the amount of recent work, many questions arising from the Wahl map are still open. We start by giving examples of families of curves of "large" dimension and nonsurjective Wahl map.

Proposition (4.1). Let $\Gamma$ be a smooth curve of genus $\gamma$ and let $\pi$ : $C \rightarrow \Gamma$ be a smooth cover of degree $n$ and genus $g$ such that either

(i) $n=2, g \geq 5 \gamma-3$ and $\pi$ has smooth ramification divisor; or

(ii) $n=3, g>\frac{15}{2} \gamma-\frac{13}{2}$ and $\pi_{*} \mathcal{O}_{C}=\mathcal{O}_{\Gamma} \oplus L^{-1} \oplus L^{-2}$, for some line bundle $L$.

Then the Wahl map $\Phi_{\omega_{C}}$ is not surjective.

Proof. In case (i) we have $\pi_{*} \mathcal{O}_{C}=\mathcal{O}_{\Gamma} \oplus L^{-1}$, for some line bundle $L$, hence $\bigwedge^{2} H^{0}\left(\omega_{C}\right) \cong \bigwedge^{2} H^{0}\left(\omega_{\Gamma} \otimes L\right) \oplus \bigwedge^{2} H^{0}\left(\omega_{\Gamma}\right) \oplus\left[H^{0}\left(\omega_{\Gamma} \otimes L\right) \otimes H^{0}\left(\omega_{\Gamma}\right)\right]$, $H^{0}\left(\omega_{C}^{3}\right) \cong H^{0}\left(\omega_{\Gamma}^{3} \otimes L^{3}\right) \oplus H^{0}\left(\omega_{\Gamma}^{3} \otimes L^{2}\right)$. It is proved by Duflot [D] that $\Phi_{\omega_{C}}$ $\operatorname{maps} \bigwedge^{2} H^{0}\left(\omega_{\Gamma} \otimes L\right) \oplus \bigwedge^{2} H^{0}\left(\omega_{\Gamma}\right)$ in $H^{0}\left(\omega_{\Gamma}^{3} \otimes L^{2}\right)$ and $V=H^{0}\left(\omega_{\Gamma} \otimes L\right) \otimes$ $H^{0}\left(\omega_{\Gamma}\right)$ in $H^{0}\left(\omega_{\Gamma}^{3} \otimes L^{3}\right)$. Note that $\operatorname{deg} L=g-2 \gamma+1$ and assume, without loss of generality, $g \geq 4$. As $H^{1}\left(\omega_{\Gamma}^{3} \otimes L\right)=H^{1}\left(\omega_{\Gamma}^{2} \otimes L\right)=0, h^{1}\left(\omega_{\Gamma}^{2} \otimes L^{-1}\right)>0$ by our hypothesis, it follows by [D, Proposition 3.4] that there is a surjection Coker $\Phi_{\left.\omega_{C}\right|_{V}} \rightarrow H^{1}\left(\omega_{\Gamma}^{2} \otimes L^{-1}\right)$, whence $\Phi_{\omega_{C}}$ is not surjective. To see (ii) let $E=L^{-1} \oplus L^{-2}$ and $R=\mathbb{P} E \stackrel{f}{\rightarrow} \Gamma$ be the corresponding ruled surface over $\Gamma$ and embed $C$ in $R$. Let $D$ be an effective divisor in the linear system $\left|\mathcal{O}_{R}(1) \otimes f^{*}(L)\right|$. We claim that

$$
\mathcal{O}_{R}(D) \otimes \mathcal{O}_{C} \cong \mathcal{O}_{C}
$$




$$
\begin{gathered}
H^{1}\left(\omega_{R}(D)\right)=0 ; \\
h^{1}\left(\omega_{C} \otimes \omega_{R}\right) \geq d-3 \gamma+3 ; \\
H^{1}\left(\Omega_{\left.R\right|_{C}}^{1} \otimes \omega_{C}^{2}\right)=0 .
\end{gathered}
$$

Let us show first that the claim implies the conclusion of (ii). By (4.2) we have a diagram

$$
\begin{array}{ccc}
\bigwedge^{2} H^{0}\left(R, \omega_{R}(C+D)\right) & \stackrel{\Phi_{\omega_{R}(C+D)}}{\longrightarrow} & H^{0}\left(R, \Omega_{R}^{1} \otimes \omega_{R}^{2}(2 C+2 D)\right) \\
\downarrow p & H^{0}\left(C, \Omega_{R \mid C}^{1} \otimes \omega_{C}^{2}\right) \\
\bigwedge^{2} H^{0}\left(C, \omega_{C}\right) & \stackrel{\Phi_{\omega_{C}}}{\longrightarrow} & H^{0}\left(C, \omega_{C}^{3}\right)
\end{array}
$$

that implies the nonsurjectivity of $\Phi_{\omega_{C}}$ since $p$ is surjective by (4.3) and $\psi$ is not surjective by (4.4) and (4.5) (in fact $d=\frac{2}{3} g-2 \gamma+\frac{4}{3}>3 \gamma-3$ ).

To see the claim let us denote by $C_{0} \in\left|\mathcal{O}_{R}(1)\right|$ a section, $r$ a fiber on $R$ and $d=\operatorname{deg} L$. We have $D \equiv C_{0}+d r, C \sim 3 C_{0}+6\left(f^{*} L\right) r$ hence $D \cdot C=0$. As $D$ is effective we get (4.2). Now $\omega_{R}(D) \cong \mathcal{O}_{R}(-1) \otimes f^{*}\left(\omega_{\Gamma} \otimes L^{-2}\right)$ whence $R^{j} f_{*}\left(\omega_{R}(D)\right)=0$ for $j=0,1$ and (4.3) follows. From the exact sequence

$$
0 \rightarrow \omega_{R}^{-1}(-C) \rightarrow \omega_{R}^{-1} \rightarrow \mathcal{O}_{C} \otimes \omega_{R}^{-1} \rightarrow 0
$$

and $R^{j} f_{*}\left(\omega_{R}^{-1}(-C)\right)=0$ for $j=0,1$ we deduce $h^{1}\left(\omega_{C} \otimes \omega_{R}\right)=h^{0}\left(\mathcal{O}_{C} \otimes\right.$ $\left.\omega_{R}^{-1}\right)=h^{0}\left(\omega_{R}^{-1}\right)=h^{0}\left(\Gamma, S^{2} E \otimes \omega_{\Gamma}^{-1} \otimes L^{3}\right) \geq h^{0}\left(\Gamma, L \otimes \omega_{\Gamma}^{-1}\right) \geq d-3 \gamma+3$, that is (4.4). As for (4.5) it is of course enough to show

$$
H^{1}\left(\Omega_{R}^{1} \otimes \omega_{R}^{2}(2 C)\right)=H^{2}\left(\Omega_{R}^{1} \otimes \omega_{R}^{2}(C)\right)=0 .
$$

To this end consider the exact sequence

$$
0 \rightarrow \mathcal{O}_{R}(-1) \otimes f^{*}\left(\omega_{\Gamma}^{3}\right) \rightarrow \Omega_{R}^{1} \otimes \omega_{R}^{2}(C) \rightarrow \mathcal{O}_{R}(-3) \otimes f^{*}\left(\omega_{\Gamma}^{2} \otimes L^{-3}\right) \rightarrow 0 .
$$

As above we have $H^{2}\left(\mathcal{O}_{R}(-1) \otimes f^{*}\left(\omega_{\Gamma}^{3}\right)\right)=0$ and also $H^{2}\left(\mathcal{O}_{R}(-3) \otimes f^{*}\left(\omega_{\Gamma}^{2} \otimes\right.\right.$ $\left.\left.L^{-3}\right)\right)=H^{0}\left(\mathcal{O}_{R}(1) \otimes f^{*}\left(\omega_{\Gamma}^{-1}\right)\right)=H^{0}\left(\Gamma, E \otimes \omega_{\Gamma}^{-1}\right)=0$. Similarly we have $H^{1}\left(\mathcal{O}_{R}(2) \otimes f^{*}\left(\omega_{\Gamma}^{3} \otimes L^{6}\right)\right)=H^{1}\left(\Gamma, S^{2} E \otimes \omega_{\Gamma}^{3} \otimes L^{6}\right)=0$ and $H^{1}\left(\mathcal{O}_{R} \otimes f^{*}\left(\omega_{\Gamma}^{2} \otimes\right.\right.$ $\left.\left.L^{3}\right)\right)=H^{1}\left(\Gamma, \omega_{\Gamma}^{2} \otimes L^{3}\right)=0$ and (4.6) is proved.

Remark (4.7). Examples of extendable canonical curves can always be obtained by taking any curve lying on the cone over a canonical curve $\Gamma$ and not meeting the vertex. Similarly for double covers of a curve. Also it 
is easily checked that the curves in example (i) of Proposition (4.1) depend on $2 g-1-\gamma$ moduli if $\gamma \geq 2(2 g-1$ if $\gamma \leq 1)$, whence (i) of Theorem (1.1) is sharp for $\alpha=1$, as 2:1 covers are extendable, by the above observation. In case (ii) of Proposition (4.1), using [Mi], we get instead $\frac{5}{3} g-3 \gamma+\frac{4}{3}$ moduli, when $\gamma \geq 2$. Even though the anticanonical divisor is effective on the surface $R$ above, we have $q \geq 2$ hence we do not know if these curves are extendable.

Open questions. (4.8) Let $\mathcal{W}_{g} \subset \mathcal{M}_{g}$ be the Wahl locus, that is the locus of curves with nonsurjective Wahl map. From Theorem (1.1) and Proposition (3.1) some natural questions arise:

(4.9) does $\mathcal{W}_{g}$ have a component of dimension larger than $\frac{3}{2} g+14$ (respectively $\frac{5}{3} g+12$ ) whose general element is not 4:1 (respectively $3: 1$ ) cover of another curve?

$\emptyset$ ?

(4.10) let $\mathrm{W}$ be a family of curves as in Proposition (3.1); is $W \cap \mathcal{W}_{g} \neq$

(4.11) is the bound of (ii), (iii) of Theorem (1.1) sharp?

Remark (4.12). The curves in Proposition (3.1) do not have maximal Clifford index, hence in particular are not Brill-Noether-Petri general. Even if (4.10) turned out to be true, they would not be counterexamples to some standard conjectures of Voisin [V2] and Wahl [W3] concerning BrillNoether-Petri general curves.

One natural family of curves to study is the Severi variety $V_{d, \delta}$ of plane curves of degree $d$ with $\delta$ nodes (as they can have many moduli). It turns out in this case that the Wahl map of a general element of $V_{d, \delta}$ is in fact surjective if $d \geq 15$ and $10 \leq \delta \leq \frac{1}{2}\left[\frac{d}{3}\right]\left(\left[\frac{d}{3}\right]+3\right)-5$ [CLM]. It is unknown what happens for higher $\delta$ (and hence for higher number of moduli).

\section{REFERENCES}

[AC] E. Arbarello and M. Cornalba, Footnotes to a paper of Beniamino Segre. The number of $g_{d}^{1}$ 's on a general d-gonal curve, and the unirationality of the Hurwitz spaces of 4-gonal and 5-gonal curves, Math. Ann., 256 (1981), 341-362.

[ACGH] E. Arbarello, M. Cornalba, P.A. Griffiths and J. Harris, Geometry of algebraic curves, Vol. I, Grundlehren der Mathematischen Wissenschaften [Fundamental Principles of Mathematical Science], 267, Springer-Verlag, New York-Berlin, 1985.

[B1] J.N. Brawner, The Gaussian-Wahl map for trigonal curves, Proc. Amer. Math. Soc., 123 (1995), 1357-1361. 
[B2] - Tetragonal curves, scrolls, and K3 surfaces., Trans. Amer. Math. Soc., 349 (1997), 3075-3091.

[CHM] C. Ciliberto, J. Harris and R. Miranda, On the surjectivity of the Wahl map, Duke Math. J., 57 (1988), 829-858.

[CLM] C. Ciliberto, A.F. Lopez and R. Miranda, On the Wahl map of plane nodal curves, In: Proceedings of the Symposium on Complex Analysis and Algebraic Geometry in memory of Michael Schneider, Bayreuth 1998, De Gruyter, Berlin-New York: 2000, 155-163.

[CM] C. Ciliberto and R. Miranda, Gaussian maps for certain families of canonical curves, In:Complex projective geometry (Trieste/Bergen, 1989), London Math. Soc. Lecture Note Ser. 179, 106-127. Cambridge Univ. Press, Cambridge, 1992.

[D] J. Duflot, Gaussian maps for double coverings, Manuscripta Math., 82 (1994), $71-87$.

[E1] D.H.J. Epema, Surfaces with canonical hyperplane sections, CWI Tract 1. Stichting Mathematisch Centrum, Centrum voor Wiskunde en Informatica, Amsterdam, 1984.

[E2] - Surfaces with canonical hyperplane sections, Nederl. Akad. Wetensch. Indag. Math., 45 (1983), 173-184.

[G] M. Green, Koszul cohomology and the geometry of projective varieties, J. Diff. Geom., 19 (1984), 125-171.

$[\mathrm{H}] \quad$ T. Horowitz, Varieties of low degree, Brown University Ph. D. Thesis, (1982) and Varieties of low $\Delta$-genus, Duke Math. J. 50 (1983), 667-683.

[L] A.F. Lopez, Surjectivity of gaussian maps on curves in $\mathbb{P}^{r}$ with general moduli, J. Algebraic Geom., 5 (1996), 609-631.

[Lv] S.M. L'vovskii, Extensions of projective varieties and deformations, Michigan Math. J., 39 (1992), 65-70.

[Ma] R. Martens, Uber den Clifford-index algebraischer kurven, J. Reine Angew. Math., 320 (1980), 68-85.

[Mi] R. Miranda, Triple covers in algebraic geometry, Amer. J. Math., 107 (1985), 1123-1158.

[P] R. Paoletti, Generalized Wahl maps and adjoint line bundles on a general curve, Pacific J. Math., 168 (1995), 313-334.

[S] F.O. Schreyer, A standard basis approach to syzygies of canonical curves, J. Reine Angew. Math., 421 (1991), 83-123.

[SD] B. Saint-Donat, Projective models of K3 surfaces, Amer. J. Math., 96 (1974), 602-639.

[V1] C. Voisin, Courbes tetragonales et cohomologie de Koszul, J. Reine Angew. Math., 387 (1988), 111-121.

[V2] - Sur l'application de Wahl des courbes satisfaisant la condition de Brill-Noether-Petri, Acta Math., 168 (1992), 249-272.

[W1] J. Wahl, The Jacobian algebra of a graded Gorenstein singularity, Duke Math. J., 55 (1987), 843-871.

[W2] — Gaussian maps on algebraic curves, J. Diff. Geom., 32 (1990), 77-98. 
[W3] On cohomology of the square of an ideal sheaf, J. Algebraic Geom., 6 (1997), 481-511.

[W4] - Hyperplane sections of Calabi-Yau varieties., preprint.

[Z] F.L. Zak, Some properties of dual varieties and their application in projective geometry, In: Algebraic Geometry, Proceedings Chicago 1989. Lecture Notes in Math. 1479. Springer, Berlin-New York: 1991, 273-280.

Ciro Ciliberto

Dipartimento di Matematica

Università di Roma "Tor Vergata"

Viale della Ricerca Scientifica

00133 Roma

Italy

cilibert@axp.mat.uniroma2.it

Angelo Felice Lopez

Dipartimento di Matematica

Università di Roma Tre

Largo San Leonardo Murialdo 1

00146 Roma

Italy

lopez@matrm3. mat . uniroma3. it 